\title{
Expression of programmed death ligand-1 predicts poor outcome in nasopharyngeal carcinoma
}

\author{
YING-FEI LI $^{1 *}$, JIAN-WU DING $^{1^{*}}$, LING-MIN LIAO ${ }^{2}$, ZHI-LIN ZHANG $^{3}$, SHOU-SHENG LIAO ${ }^{4}$, \\ YING WU ${ }^{1}$, DAN-YANG ZHOU ${ }^{1}$, AN-WEN LIU ${ }^{1}$ and LONG HUANG ${ }^{1}$ \\ Departments of ${ }^{1}$ Oncology, ${ }^{2}$ Ultrasound, ${ }^{3}$ Otorhinolaryngology and ${ }^{4}$ Pathology, \\ The Second Affiliated Hospital of Nanchang University, Nanchang, Jiangxi, P.R. China
}

Received October 17, 2016; Accepted May 15, 2017

DOI: $10.3892 / \mathrm{mco} .2017 .1318$

\begin{abstract}
Programmed death ligand-1 (PD-L1) is a potentially important tumor immunotherapy target. However, whether PD-L1 expression is associated with survival in nasopharyngeal carcinoma (NPC) remains controversial. The aim of the present study was to investigate the association between PD-L1 expression and prognosis in NPC. The expression of PD-L1 was assessed in tumor specimens from 120 patients with NPC using immunohistochemistry. Staining was evaluated using the $\mathrm{H}$-score method. The associations between PD-L1 expression and clinical characteristics and prognosis were analyzed. Overall, $78 \%$ of the patients had stage I-III and $22 \%$ had stage IV disease. The estimated 5-year overall survival (OS) and disease-free survival (DFS) rates for the entire cohort were 87.5 and $70.1 \%$, respectively. PD-L1 expression was detected in $85(71 \%)$ patients and was localized to the tumor cells. High tumor expression of PD-L1 (median $\mathrm{H}$-score $\geq 5$ ) was associated with significantly poorer OS $(\mathrm{P}=0.023)$ and DFS $(\mathrm{P}=0.002)$. Univariate analysis indicated that low PD-L1 expression was associated with better DFS compared with high PD-L1 expression $(\mathrm{HR}=0.163,95 \% \mathrm{CI}$ : $0.044-0.600, \mathrm{P}=0.006$ for DFS). Multivariate analysis revealed that $\mathrm{T}$ stage $(\mathrm{HR}=8.190,95 \% \mathrm{CI}: 1.355-18.152 ; \mathrm{P}=0.023)$ and PD-L1 expression level $(\mathrm{HR}=0.124,95 \% \mathrm{CI}: 0.031-0.509$; $\mathrm{P}=0.001)$ served as independent prognostic factors for DFS. In conclusion, tumor PD-L1 expression was found to be a significant prognostic factor in NPC, and high PD-L1 expression may be of prognostic value for recurrence and metastasis following conventional treatments.
\end{abstract}

Correspondence to: Dr Long Huang or Professor An-Wen Liu, Department of Oncology, The Second Affiliated Hospital of Nanchang University, 1 Minde Road, Nanchang, Jiangxi 330000, P.R. China

E-mail: huanglongdoctor@163.com

E-mail: awliu666@163.com

*Contributed equally

Key words: nasopharyngeal carcinoma, programmed death ligand-1, expression, prognosis

\section{Introduction}

Nasopharyngeal carcinoma (NPC) is disproportionately common in southern China (1), where the annual incidence is $15-50 / 100,000$ individuals (2). Radiotherapy (RT) is the primary treatment for NPC. While advances in tumor imaging and RT technologies have increased 5-year disease-free survival (DFS) to $77 \%$ for early-stage disease (3), distant metastasis remains a major obstacle to further improvements in survival (4).

The immune checkpoints proteins programmed death-1 (PD-1) and programmed death ligand-1 (PD-L1) have been reported to be important immunotherapy targets. The antitumor efficacy of blocking these targets has been confirmed in vitro as well as in vivo. Blocking the PD-1/PD-L1 signaling pathway represents a promising immunotherapeutic strategy to enhance the ability of the immune system to target cancer cells $(5,6)$.

PD-L1 is expressed in a variety of tumor types, including esophageal, gastrointestinal tract, pancreatic, breast, lung and kidney carcinomas $(7,8)$. Several studies have indicated that tumor PD-L1 expression is associated with poor prognosis $(9,10)$; however, it remains unclear whether such an association exists in NPC. The aim of the present study was to investigate the associations between tumor PD-L1 expression and prognosis in NPC.

\section{Patients and methods}

Patients and sample collection. Two cohorts of patients with NPC were enrolled: 62 patients with primary biopsy-confirmed NPC diagnosed between January 2009 and January 2012 at the Second Affiliated Hospital of Nanchang University (Nanchang, China) and 58 patients with primary biopsy-confirmed NPC diagnosed between January 2010 and May 2015 at the People's Hospital of Fuzhou (Fuzhou, China). Formalin-fixed paraffin-embedded tissues were obtained from the initial diagnostic biopsy specimens. Information on the clinical course of each patient, including sex, age, tumor staging and smoking history, was obtained from medical records. Complete follow-up data were available for all 62 patients treated at the Second Affiliated Hospital of Nanchang University. Data on disease and vital status for these patients were obtained using 
a prospectively-maintained hospital tumor registry. The study protocol was approved by the Medical Ethics Committees of the Second Affiliated Hospital of Nanchang University and the People's Hospital of Fuzhou.

Immunohistochemistry. Immunohistochemical staining for the PD-L1 protein was performed using sections prepared from the formalin-fixed diagnostic samples. Briefly, 4- $\mu \mathrm{m}$ sections were deparaffinized in xylene and rehydrated through a graded ethanol series to distilled water. After processing via routine procedures, the sections were incubated overnight at $4^{\circ} \mathrm{C}$ with rabbit anti-PD-L1 antibody (E1L3 $\mathrm{N}^{\text {TM }}$, catalog no. 13684, Cell Signaling Technology Inc., Shanghai, China) diluted 1:100 in blocking solution, followed by incubation with a horseradish peroxidase-conjugated secondary antibody (MaxVision Immunohistochemical Detection kit, rabbit/mouse, 1:100 dilution, catalog no. 5001, MaxVision Biosciences, Inc., Fuzhou, China) according to the manufacturer's instructions. After washing, color was developed by incubation in 3,3'-diaminobenzidine tetrahydrochloride, followed by hematoxylin counterstaining.

The staining was independently assessed by two surgical pathologists using a semi-quantitative scale that ranges from 0 to $100 \%$ for the proportion of PD-L1-positive cancer cells. The mean score for replicate samples was recorded. Two groups of patients were created based on receiver operating characteristic (ROC) curves. According to the results, 20\% was set as the cut-off value for dividing the samples into high PD-L1 and low PD-L1 group for DFS. The areas under the ROC curves were 0.827 .

Statistical analysis. The associations between PD-L1 expression and clinicopathological characteristics were assessed using Pearson's $\chi^{2}$ test. Overall survival (OS) and DFS were compared using the Kaplan-Meier method and log-rank tests. Cox proportional hazards analysis was performed to calculate hazard ratios (HRs) and 95\% confidence intervals (CIs) to evaluate the associations between tumor PD-L1 expression and survival outcome. Multivariate survival analysis was conducted for all of the parameters using the Cox regression model. All analyses were performed using SPSS software, version 13.0 (SPSS Inc., Chicago, IL, USA). Two-sided P-values $<0.05$ were considered to indicate statistically significant differences.

\section{Results}

Clinicopathological characteristics. The 120 patients in this cohort ranged in age from 17 to 69 years (median, 48 years). A total of $78 \%(79 / 102)$ of the patients had stage I-III and $22 \%$ (23/102) had stage IV NPC according to the 1997 American Joint Committee on Cancer staging system (11). All the cases were classified as undifferentiated non-keratinizing NPC. The estimated 5-year OS and DFS rates for the group eligible for survival analysis (the 62 patients treated at the Second Affiliated Hospital of Nanchang University) were 87.5 and $70.1 \%$, respectively. Other clinicopathological characteristics are summarized in Table I.

PD-L1 protein is frequently expressed in NPC. To investigate whether PD-L1 is expressed in NPC similar to its expression
Table I. Associations between PD-L1 expression and the clinicopathological characteristics of nasopharyngeal carcinoma.

\begin{tabular}{|c|c|c|c|}
\hline \multirow{2}{*}{$\begin{array}{l}\text { Clinicopathological } \\
\text { characteristics }\end{array}$} & \multicolumn{2}{|c|}{ PD-L1 expression } & \multirow[b]{2}{*}{ P-value } \\
\hline & High & Low & \\
\hline \multicolumn{4}{|l|}{ Sex } \\
\hline Male & 40 & 46 & \\
\hline Female & 14 & 20 & 0.597 \\
\hline \multicolumn{4}{|l|}{ Age, years } \\
\hline$\leq 45$ & 9 & 26 & \\
\hline$>45$ & 45 & 40 & 0.006 \\
\hline \multicolumn{4}{|l|}{ Smoking history } \\
\hline No & 26 & 28 & \\
\hline Yes & 18 & 19 & 0.963 \\
\hline \multicolumn{4}{|l|}{ Histology } \\
\hline Differentiated & 29 & 26 & \\
\hline Undifferentiated & 25 & 40 & 0.118 \\
\hline \multicolumn{4}{|l|}{ AJCC stage (2010) } \\
\hline I-III & 32 & 47 & \\
\hline IV & 15 & 8 & 0.036 \\
\hline \multicolumn{4}{|l|}{ T stage } \\
\hline $\mathrm{T} 1 / \mathrm{T} 2$ & 17 & 30 & \\
\hline T3/T4 & 30 & 25 & 0.063 \\
\hline \multicolumn{4}{|l|}{ N stage } \\
\hline N0/N1 & 22 & 17 & \\
\hline $\mathrm{N} 2 / \mathrm{N} 3$ & 25 & 38 & 0.100 \\
\hline
\end{tabular}

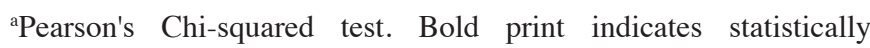
significant values. AJCC, American Joint Committee on Cancer; PD-L1, programmed death ligand-1.

in other tumors, PD-L1 protein expression was assessed using immunohistochemistry in tumor samples from 120 patients with NPC. The PD-L1 protein was found to be frequently expressed in NPC. PD-L1 expression was detected in $71 \%$ $(85 / 120)$ of the tumor samples. The PD-L1 protein was localized to the cell membrane of the primary tumor cells (Fig. 1A) and metastatic lymph nodes (Fig. 1D). Moreover, PD-L1 protein expression was significantly higher in the tumor cells compared with that in adjacent non-cancerous tissues (Fig. 1B and C).

Associations between PD-L1 expression and the clinicopathological characteristics of NPC. To identify the clinical relevance of PD-L1 expression in NPC, the associations between PD-L1 expression and clinicopathological characteristics of NPC, such as sex, age, smoking history, histology and TNM stage, were examined. The 120 patients were further classified into the high PD-L1 (n=54) and low PD-L1 $(n=66)$ groups based on the tumor PD-L1 expression level. The results revealed that high PD-L1 expression was significantly associated with age $(\mathrm{P}=0.006)$ and stage $(\mathrm{P}=0.036)$, but not the other clinical characteristics examined (Table I). 
Table II. Univariate analysis of the associations between the clinicopathological characteristics of nasopharyngeal carcinoma and disease-free survival.

\begin{tabular}{lccr}
\hline Clinicopathological characteristics & Subset & Hazard ratio (95\% CI) & P-value \\
\hline Gender & Male vs. female & $1.322(0.431-4.056)$ & 0.626 \\
Age, years & $>45$ vs. $\leq 45$ & $1.747(0.570-5.352)$ & 0.329 \\
Smoking history & No vs. yes & $0.785(0.207-2.973)$ & 0.722 \\
Histology & Undifferentiated vs. differentiated & $1.595(0.521-4.886)$ & 0.413 \\
AJCC stage & IV vs. I-III & $1.662(0.533-5.181)$ & 0.381 \\
T stage & T3/T4 vs. T1/T2 & $4.081(1.130-18.14)$ & $\mathbf{0 . 0 4 8}$ \\
N stage & N0/N1 vs. S N2/N3 & $0.530(0.145-1.947)$ & 0.339 \\
Neoadjuvant chemotherapy 1 & TPF vs. TP/FP/other & $0.489(0.134-1.792)$ & 0.281 \\
Neoadjuvant chemotherapy 2 & Includes NDP vs. DDP & $1.669(0.451-6.181)$ & 0.443 \\
Neoadjuvant chemotherapy 3 & Yes vs. no & $0.832(0.270-2.562)$ & 0.749 \\
Radiotherapy & CCRT vs. RT & $0.461(0.149-1.426)$ & 0.179 \\
Adjuvant chemotherapy & 2-3 vs. 0-1 cycles & $0.370(0.113-1.218)$ & 0.102 \\
PD-L1 expression & Negative vs. positive & $0.163(0.044-0.600)$ & $\mathbf{0 . 0 0 6}$ \\
\hline
\end{tabular}

Bold print indicates statistically significant values. AJCC, American Joint Committee on Cancer; CI, confidence interval; TPF, docetaxel + platinum + fluorouracil; TP, docetaxel + platinum; FP, fluorouracil + platinum; NDP, nedaplatin; DDP, cisplatin; CCRT, concurrent chemoradiotherapy; RT, radiotherapy; PD-L1, programmed death ligand-1.
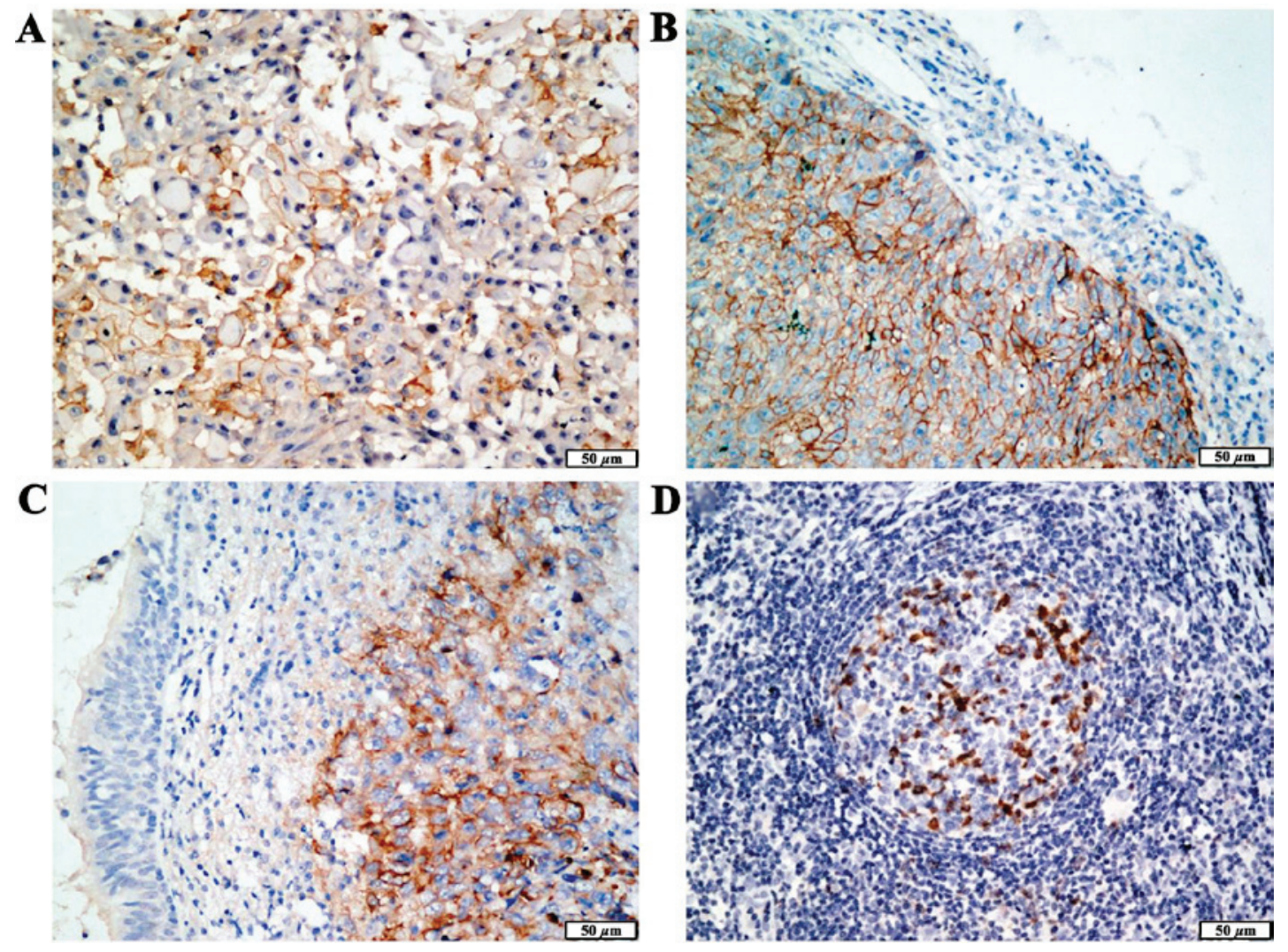

Figure 1. Representative images of immunohistochemical staining for programmed death ligand 1 (PD-L1) in nasopharyngeal carcinoma (NPC). (A) PD-L1 was expressed in the cell membrane of the primary tumor cells (magnification, x40). (B and C) The PD-L1 protein was expressed in NPC cells, but not the surrounding normal nasopharyngeal epithelial cells (magnification, x40). (D) PD-L1 was expressed in the germinal center of lymph nodes (magnification, $\mathrm{x} 40$ ).

Associations between PD-L1 expression and prognosis in $N P C$. Estimated 5-year OS and DFS for the group eligible for survival analysis (the 62 patients treated at Second
Affiliated Hospital of Nanchang University) were 87.5 and $70.1 \%$, respectively (Fig. $2 \mathrm{~A}$ and B). The OS and DFS curves for the high PD-L1 and low PD-L1 groups are shown 
A

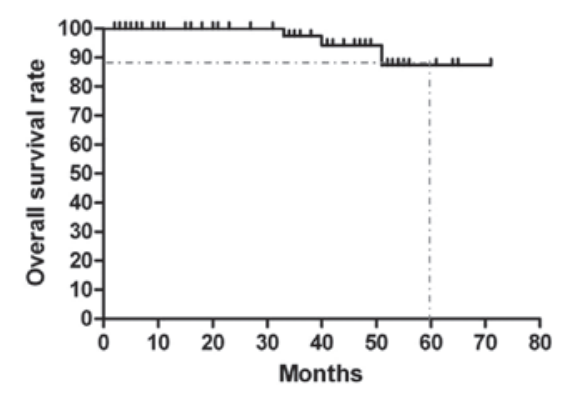

C

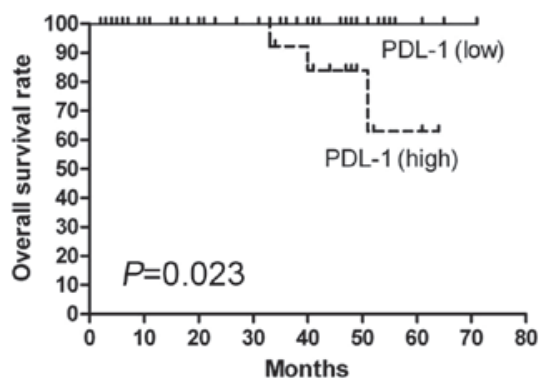

B

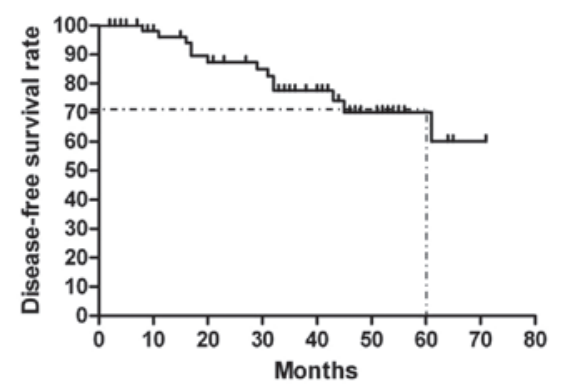

D

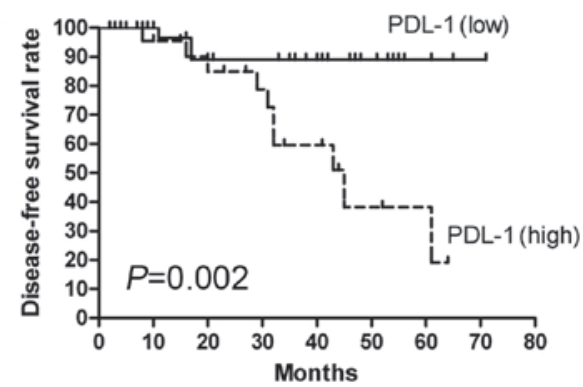

Figure 2. Kaplan-Meier overall survival (OS) and disease-free survival (DFS) curves for the 62 patients eligible for survival analysis. (A) OS and (B) DFS curves for the 62 patients. (C) OS and (D) DFS curves for patients stratified by high and low PD-L1 expression. PD-L1, programmed death ligand 1.

in Fig. 2C and D. As expected, patients with high PD-L1 expression had significantly poorer OS $(\mathrm{P}=0.023)$ and $\mathrm{DFS}$ $(\mathrm{P}=0.002)$ compared with patients with low PD-L1 expression. Univariate Cox proportional regression analysis revealed that $\mathrm{T}$ stage $(\mathrm{HR}=4.081,95 \% \mathrm{CI}: 1.130-18.14 ; \mathrm{P}=0.048)$ and PD-L1 expression $(\mathrm{HR}=0.163,95 \%$ CI: 0.044-0.600; $\mathrm{P}=0.006)$ were prognostic factors for DFS (Table II). A multivariate analysis using the Cox regression model revealed that $\mathrm{T}$ stage $(\mathrm{HR}=8.190,95 \% \mathrm{CI}: 1.355-18.152 ; \mathrm{P}=0.023)$ and $\mathrm{PD}-\mathrm{L} 1$ expression level $(\mathrm{HR}=0.124,95 \% \mathrm{CI}$ : 0.031-0.509; $\mathrm{P}=0.001)$ served as independent prognostic factors for DFS.

\section{Discussion}

Tumor immune evasion is considered to be a hallmark of cancer (12). Blockade of immune checkpoints represents a promising therapeutic approach to activate antitumor immunity (13). PD-L1 is recognized as an important immunosuppressive factor (14) and is upregulated in a variety of Epstein-Barr virus (EBV)-associated malignancies (15), although its expression in NPC, an EBV-associated malignancy with a higher metastatic potential compared with other head and neck cancers, is poorly characterized $(16,17)$. The expression of PD-L1 is associated with the treatment response to nivolumab, an anti-PD-1 antibody, in a subset of tumor types (18). The efficacy of immune-targeted therapies in EBV-associated malignancies requires further investigation. Therefore, assessing the expression of biomarkers such as PD-L1 may help identify patients who will benefit from immunomodulatory agents. However, variation in PD-L1 expression rates previously observed in a study of various cancers has been attributed to differences in cut-off values, antibodies, and study populations $(19,20)$. In lung cancer characterized by
PD-L1 expression, the expression rates varied from 24 to $60 \%$ using the same 5\% cut-off value $(21,22)$, and from 21 to $95 \%$ according to different cut-off values of 1,10 and $50 \%(23,24)$. Thus, the use of PD-L1 expression as a marker remains controversial due to the different antibodies used, the method of assessment and the different thresholds.

In the present study, tumor PD-L1 expression and its association with the clinicopathological characteristics and survival outcomes of NPC were analyzed. The PD-L1 protein was found to be frequently expressed in NPC and high tumor expression of PD-L1 was prognostic for poor DFS. Fang et al (25) reported that 89\% (16/18) of EBV-associated NPC cases expressed PD-L1 in the malignant tumor cells, which is consistent with the findings of our study. Additionally, high expression of PD-L1 was also found to be associated with poor clinical prognosis in renal cell carcinoma, hepatocellular cancer and breast cancer (26-29).

Previous studies demonstrated that constitutively-activated oncogenic pathways may upregulate PD-L1. Parsa et al reported loss of phosphatase and tensin homolog and resulting activation of the phosphoinositide 3-kinase pathway significantly upregulates PD-L1 in glioma (30). Marzec et al found constitutive activation of nucleophosmin/anaplastic lymphoma kinase induces PD-L1 expression via signal transducer and activator of transcription 3 (31). The mechanism through which PD-L1 is upregulated in NPC has not been fully elucidated. Fang et al observed that high levels of PD-L1 expression in EBV-infected NPC cells were associated with latent membrane protein 1 (LMP1)-mediated oncogenic pathways and immune modulation via excretion of interferon- $\gamma(25)$. These results indicated that inhibition of the LMP1 oncogenic pathway and PD-1/PD-L1 checkpoints may provide a clinical benefit during the treatment of EBV-associated NPC. 
In conclusion, PD-L1 is frequently upregulated in NPC, and high PD-L1 expression was associated with poor prognosis. These findings implicate PD-L1 in the development of NPC and highlight the potential of PD-L1 as a therapeutic target for NPC. Patients with NPC whose tumors express high levels of PD-L1 may be optimal candidates for clinical trials of strategies aiming to block PD-L1.

\section{Acknowledgements}

This study was supported by the National Natural Science Foundation of China (grant no. 81460393), the Natural Science Foundation of Jiangxi Province, China (grant nos. 20142BAB215039 and 20151BAB215020), the Project of Jiangxi Province Science and Technology Plan (grant nos. 20141BBG70041 and GJJ14059), the Youth Science Fund Project of the Second Affiliated Hospital of Nanchang University (grant no. 2014YNQN12004 to Long Huang), and in part by the Natural Science Foundation of China (grant no. 81460449 to Ling-Min Liao).

\section{References}

1. Licitra L, Bernier J, Cvitkovic E, Grandi C, Spinazzé S, Bruzzi P, Gatta G and Molinari R: Cancer of the nasopharynx. Crit Rev Oncol Hematol 45: 199-213, 2003.

2. Cao SM, Simons MJ and Qian CN: The prevalence and prevention of nasopharyngeal carcinoma in China. Chin J Cancer 30: 114-119, 2011.

3. Ma BB, Hui EP and Chan AT: Systemic approach to improving treatment outcome in nasopharyngeal carcinoma: Current and future directions. Cancer Sci 99: 1311-1318, 2008.

4. Xiao WW, Huang SM, Han F, Wu SX, Lu LX, Lin CG, Deng XW, Lu TX, Cui NJ and Zhao C: Local control, survival, and late toxicities of locally advanced nasopharyngeal carcinoma treated by simultaneous modulated accelerated radiotherapy combined with cisplatin concurrent chemotherapy: Long-term results of a phase 2 study. Cancer 117: 1874-1883, 2011.

5. Topalian SL, Drake CG and Pardoll DM: Targeting the PD-1/B7-H1(PD-L1) pathway to activate anti-tumor immunity. Curr Opin Immunol 24: 207-212, 2012.

6. Lu J, Lee-Gabel L, Nadeau MC, Ferencz TM and Soefje SA: Clinical evaluation of compounds targeting PD-1/PD-L1 pathway for cancer immunotherapy. J Oncol Pharm Pract 21: 451-467, 2015.

7. Zou W and Chen L: Inhibitory B7-family molecules in the tumour microenvironment. Nat Rev Immunol 8: 467-477, 2008.

8. Flies DB and Chen L: The new B7s: Playing a pivotal role in tumor immunity. J Immunother 30: 251-260, 2007.

9. Muenst S, Schaerli AR, Gao F, Däster S, Trella E, Droeser RA, Muraro MG, Zajac P, Zanetti R, Gillanders WE, et al: Expression of programmed death ligand 1 (PD-L1) is associated with poor prognosis in human breast cancer. Breast Cancer Res Treat 146: 15-24, 2014.

10. Thompson RH, Kuntz SM, Leibovich BC, Dong H, Lohse CM, Webster WS, Sengupta S, Frank I, Parker AS, Zincke H, et al: Tumor B7-H1 is associated with poor prognosis in renal cell carcinoma patients with long-term follow-up. Cancer Res 66: 3381-3385, 2006.

11. Chua DT, Sham JS, Wei WI, Ho WK and Au GK: The predictive value of the 1997 American joint committee on cancer stage classification in determining failure patterns in nasopharyngeal carcinoma. Cancer 92: 2845-2855, 2001.

12. Hanahan D and Weinberg RA: Hallmarks of cancer: The next generation. Cell 144: 646-674, 2011.

13. Pardoll DM: The blockade of immune checkpoints in cancer immunotherapy. Nat Rev Cancer 12: 252-264, 2012.

14. Dolan DE and Gupta S: PD-1 pathway inhibitors: Changing he landscape of cancer immunotherapy. Cancer Control 21: 231-237, 2014.
15. Chen BJ, Chapuy B, Ouyang J, Sun HH, Roemer MG, Xu ML, Yu H, Fletcher CD, Freeman GJ, Shipp MA and Rodig SJ: PD-L1 expression is characteristic of a subset of aggressive B-cell lymphomas and virus-associated malignancies. Clin Cancer Res 19: 3462-3473, 2013.

16. Lin X, Gudgeon NH, Hui EP, Jia H, Qun X, Taylor GS Barnardo MC, Lin CK, Rickinson AB and Chan AT: CD4 and CD8 T cell responses to tumour-associated Epstein-Barr virus antigens in nasopharyngeal carcinoma patients. Cancer Immunol Immunother 57: 963-975, 2008.

17. Li HP, Peng CC, Chung IC, Huang MY, Huang ST, Chen CC, Chang KP, Hsu CL and Chang YS: Aberrantly hypermethylated Homeobox A2 derepresses metallo proteinase-9 through TBP and promotes invasion in Nasopharyngeal carcinoma. Oncotarget 4: 2154-2165, 2013.

18. Topalian SL, Hodi FS, Brahmer JR, Gettinger SN, Smith DC, McDermott DF, Powderly JD, Carvajal RD, Sosman JA, Atkins MB, et al: Safety, activity, and immune correlates of anti-PD-1 antibody in cancer. N Engl J Med 366: 2443-2454, 2012.

19. Teixido C,Karachaliou N, González-Cao M, Morales-Espinosa D and Rosell R: Assays for predicting and monitoring responses to lung cancer immunotherapy. Cancer Biol Med 12: 87-95, 2015.

20. Patel SP and Kurzrock R: PD-L1 expression as a predictive biomarker in cancer immunotherapy. Mol Cancer Ther 14: 847-856, 2015.

21. D'Incecco A, Andreozzi M, Ludovini V, Rossi E, Capodanno A, Landi L, Tibaldi C, Minuti G, Salvini J, Coppi E, et al: PD-1 and PD-L1 expression in molecularly selected non-small-cell lung cancer patients. Br J Cancer 112: 95-102, 2015.

22. Herbst RS, Soria JC, Kowanetz M, Fine GD, Hamid O, Gordon MS, Sosman JA, McDermott DF, Powderly JD, Gettinger SN, et al: Predictive correlates of response to the anti-PD-L1 antibody MPDL3280A in cancer patients. Nature 515: 563-567, 2014.

23. Dong H, Strome SE, Salomao DR, Tamura H, Hirano F, Flies DB, Roche PC, Lu J, Zhu G, Tamada K, et al: Tumor-associated B7-H1 promotes T-cell apoptosis: A potential mechanism of immune evasion. Nat Med 8: 793-800, 2002.

24. Garon EB, Rizvi NA, Hui R, Leighl N, Balmanoukian AS, Eder JP, Patnaik A, Aggarwal C, Gubens M, Horn L, et al: Pembrolizumab for the treatment of non-small-cell lung cancer. N Engl J Med 372: 2018-2028, 2015.

25. Fang W, Zhang J, Hong S, Zhan J, Chen N, Qin T, Tang Y, Zhang Y, Kang S, Zhou T, et al: EBV-driven LMP1 and IFN- $\gamma$ up-regulate PD-L1 in nasopharyngeal carcinoma: Implications for oncotargeted therapy. Oncotarget 5: 12189-12202, 2014.

26. Thompson RH, Dong H and Kwon ED: Implications of B7-H1 expression in clear cell carcinoma of the kidney for prognostication and therapy. Clin Cancer Res 13: 709s-715s, 2007.

27. Shi F, Shi M, Zeng Z, Qi RZ, Liu ZW, Zhang JY, Yang YP, Tien P and Wang FS: PD-1 and PD-L1 upregulation promotes CD8(+) T-cell apoptosis and postoperative recurrence in hepatocellular carcinoma patients. Int J Cancer 128: 887-896, 2011.

28. Ghebeh H, Tulbah A, Mohammed S, Elkum N, Bin Amer SM, Al-Tweigeri T and Dermime S: Expression of B7-H1 in breast cancer patients is strongly associated with high proliferative Ki-67-expressing tumor cells. Int J Cancer 121: 751-758, 2007.

29. Muenst S, Soysal SD, Gao F, Obermann EC, Oertli D and Gillanders WE: The presence of programmed death 1 (PD-1)-positive tumor-infiltrating lymphocytes is associated with poor prognosis in human breast cancer. Breast Cancer Res Treat 139: 667-676, 2013.

30. Parsa AT, Waldron JS, Panner A, Crane CA, Parney IF, Barry JJ, Cachola KE, Murray JC, Tihan T, Jensen MC, et al: Loss of tumor suppressor PTEN function increases B7-H1 expression and immunoresistance in glioma. Nat Med 13: 84-88, 2007.

31. Marzec M,Zhang Q, Goradia A, Raghunath PN,Liu X,Paessler M, Wang HY, Wysocka M, Cheng M, Ruggeri BA and Wasik MA: Oncogenic kinase NPM/ALK induces through STAT3 expression of immunosuppressive protein CD274 (PD-L1, B7-H1). Proc Natl Acad Sci USA 105: 20852-20857, 2008. 